\title{
A systematic review of nonsynonymous single nucleotide polymorphisms in the renin-angiotensin-aldosterone system
}

\author{
Tomasz Rechciński®, Jarosław D. Kasprzak® \\ $1^{\text {st }}$ Department and Chair of Cardiology, Medical University of Lodz, Poland
}

\begin{abstract}
In this recent publication review the authors aimed to collect evidence of impact of nonsynonymous single nucleotide polymorphisms (nsSNP) in the renin-angiotensin-aldosterone system on patients' phenotype not only regarding arterial hypertension and its complications, but also the impact on other diseases of interest outside the field of cardiovascular medicine.

PubMed database records published between 2017-2020 were searched and all positive case-control studies or positive studies with human DNA were selected.

The search identified 104 articles, of which 22 were included on the basis of the inclusion criteria. This paper presents the impact of 44 nsSNPs in panels for genes of renin, angiotensinogen, angiotensin-converting enzyme, angiotensin receptor and aldosterone on the clinical picture of investigated cohorts or on the peptide-protein interactions as consequence of nsSNPs.

Genetic variability in nsSNPs of the RAAS is involved in the pathogenesis of arterial hypertension and its complications, and surprisingly also in the pathogenesis of conditions not associated with elevated blood pressure, like neoplasms or inflammatory diseases. (Cardiol J 2022; 29, 6: 1020-1027)
\end{abstract}

Key words: nonsynonymous single nucleotide polymorphisms, renin-angiotensin-aldosterone system

\section{Introduction}

The renin-angiotensin-aldosterone system (RAAS) is strongly involved in the pathogenesis of arterial hypertension, as well as in the occurrence of its complications. The diversity of the clinical course of this disease and the acceleration of target organ damage may depend not only on the presence of concomitant diseases, on environmental factors including lifestyle, quality of health care, utilization and adherence to proven preventive therapies, but also to some extent on genetic factors. The rapid development of genetics shows that nonsynonymous single nucleotide polymorphisms (SNP) are one of many types of human genome variability, which along with changes in epigenetic modulators (e.g., microRNA) or posttranscriptional modifications (e.g., methylation of DNA), play a role in shaping the final picture of the disease. Although transcriptional errors which lead to a single nucleotide swap from a wild to a mutated variant usually have a spontaneous nature, they may be also inherited by descendants and be of importance even in fetal life. In 2017 it was suggested that the role of polymorphisms in the RAAS assessed in various population studies was overestimated during the "candidate gene era" and that there is a need to reconsider the clinical significance of RAAS variants for individuals in the framework of "precision medicine" [1]. Surprisingly, in the review prepared by Ji et al. [1], genetic variants of some genes related to the RAAS were not associ-

Address for correspondence: Tomasz Rechciński, MD, PhD, FESC, $1^{\text {st }}$ Department and Chair of Cardiology, Medical University of Lodz, W1. Biegański W.S.S. in Lodz, ul. Kniaziewicza 1/5, 91-347 Łódź, Poland, tel: +48 422516216 , fax: +48 42 2516015, e-mail: tomasz.rechcinski@office365.umed.pl

Received: 30.12.2020 Accepted: 5.05.2021 Early publication date: 28.05.2021

This article is available in open access under Creative Common Attribution-Non-Commercial-No Derivatives 4.0 International (CC BY-NC-ND 4.0) license, allowing to download articles and share them with others as long as they credit the authors and the publisher, but without permission to change them in any way or use them commercially. 
ated with risk of cardiovascular diseases, but rather with abnormal thyroid function, schizophrenia, lead poisoning, fibrosis, angiotensin-convertingenzyme (ACE) induced cough or with lipoprotein concentrations. Additionally, some new technologies enabled using artificial intelligence for the prediction of SNPs importance with regard to the interactions of their products (protein-peptide) and, potentially - for their altered function [2]. The aim of this study was to critically review the results of studies on the most investigated RAAS SNPs published since 2017 to date.

\section{Methods}

Two reviewers working independently extracted from PubMed database (www.pubmed.ncbi. nlm.nih.gov) the relevant publications released between January 1, 2017 and November 30, 2020. Studies with the following keywords (as inclusion criteria): SNP, RAAS, renin, angiotensinogen, angiotensin, aldosterone, angiotensin-converting enzyme, angiotensin-receptor were extracted. Reviews, meta-analyses, case reports, basic science studies with experimental medicinal compounds and studies performed in unique or specific populations were excluded. Only English-language, positive human studies or studies with human DNA were selected for this review of the latest reports on SNP in the RAAS. Figure 1 with flow-chart shows the selection of papers qualified for the present publication.

In the first step, two authors reviewed the abstracts and excluded those not meeting eligibility criteria. The articles were included after two investigators independently recognized the article as eligible to this review. Differences between two reviewers were resolved by consensus. Twenty-two articles referring to 44 SNPs were finally chosen for inclusion in this review - these SNPs are presented on the Central illustration.

\section{Results}

\section{The renin panel}

The resequencing study conducted among the 1906 participants of the GenSalt study aimed to find SNP association with salt-sensitive arterial hypertension in a community from the Han Chinese population with a habitually high $\mathrm{Na}$-intake from rural areas [3]. Gene-based analyses were performed in 300 participants selected with the highest arterial pressure dietary response (mean

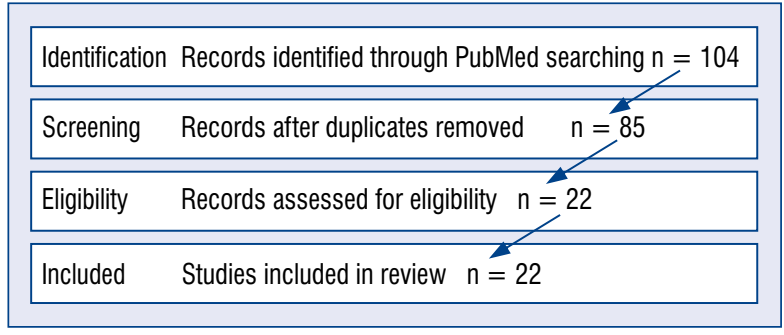

Figure 1. Flow diagram of publications selection.

$+11.8 \mathrm{mmHg}$ ) and 300 with the lowest response (mean $-1.1 \mathrm{mmHg}$ ). The probands in salt-sensitive group had higher mean age (41.6 vs. 38.2 years) than salt-resistant probands, and the rate of females was higher (52.5\% vs. 35.6\%). Seven RAAS genes with potential biological effect on blood pressure regulation were selected - renin-binding protein gene $(R E N B P)$, angiotensin I converting enzyme 2 gene $(A C E 2)$ and angiotensin II type 1 receptor gene $(A G T R 1)$ were in this number. Within 50 single-nucleotide variants only low-frequency, missense SNP in RENBP gene described as rs78377269, present in exonic region of chromosome X (C to A substitution in 153941584 position) reached statistical significance in a single marker analysis $(\mathrm{p}=0.03)$ after adjustment for multiple testing when studied subgroups were compared. Each copy of the minor allele corresponded to a $1.63 \mathrm{mmHg}$ larger mean arterial pressure response to dietary sodium intervention and a 2.21-fold increased odds of salt sensitivity (95\% confidence interval [CI] 1.10-4.42). The authors of that study do not exclude that the other SNPs tested in the study may explain, in aggregate, the phenomenon of salt-response hypertension.

Since renin is associated with preeclampsia and eclampsia - genetic variability of its gene located on chromosome 1 , was a subject of studies with mothers and their offspring, as well as mothers alone in this respect in Chinese and Romanian centers, respectively.

Three SNPs were analyzed by Yu et al. [4] in a study which included 347 preeclampsia/eclampsia patients and 700 controls. The fetal heterozygotic genotype rs5707 in renin gene REN (AC) was significantly $(\mathrm{p}=0.004)$ associated with an increased risk of preeclampsia/eclampsia when accompanied by a mother's body mass index $\geq 24 \mathrm{~kg} / \mathrm{m}^{2}$ — odds ratio (OR) 2.75 (95\% CI 1.5-5.06) [4]. In a study by Procopciuc et al. [5] where 87 pregnant women with preeclampsia were compared with 130 con- 


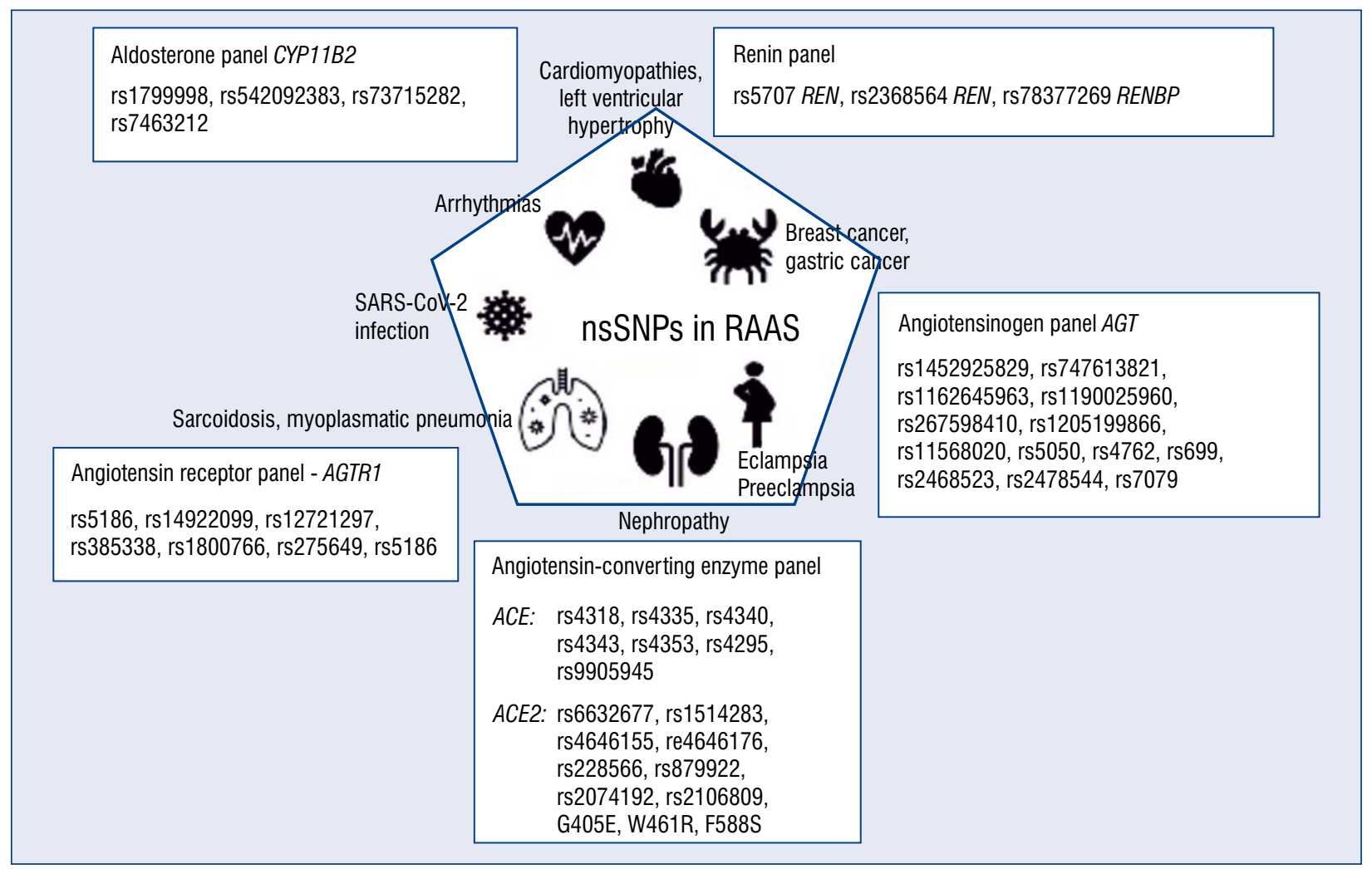

Central illustration. The nonsynonymous single-nucleotide polymorphisms (nsSNPs) and spectrum of diseases described in this review; ACE - angiotensin converting enzyme; RAAS — renin-angiotensin-aldosterone system; SARS-CoV-2 - severe acute respiratory syndrome coronavirus 2 .

trols also SNP rs2368564 (G83A) in REN with a heterozygotic form were significantly associated $(p=0.009)$ with late-onset preeclampsia [5].

\section{The angiotensinogen panel}

A different approach to genetic variability evaluation was applied in a study by Goswami [6] regarding angiotensinogen gene $(A G T)$. That author validated 354 SNPs of $A G T$ and determined their conservation degree in a 9 -step scale. Using a computational modeling, 3-dimentional structures of wild-type and mutant $A G T$ variants were generated. The nature of each of the 485 amino acids of this macromolecule was defined as exposed or as buried depending on what extent it is hidden in the protein structure space, moreover their functional or structural role for the protein was predicted. Three SNPs (rs1452925829, rs746613821 and rs1162645963) were identified as being highly destabilizing for renin-angiotensinogen binding, they had also strongest impact on the change of the interface area between renin and angiotensinogen. It was proved earlier that a majority of diseases associated with nonsynonymous SNPs are caused by the instability of the proteins. The highest scores (0.808-0.919) for the prediction of the structure, function and post-translational modifications of human angiotensinogen were observed for rs1190025960, rs267598410, and rs1205199806 which lead to following swaps of amino acids in the indicated positions: G149R, R477G, and L162P, respectively. Substitutions of amino acids cause changes in some physical properties of angiotensinogen such as electrostatic potentials, nonpolar and polar solvation energy, mechanical energy and van der Waals forces. After $A G T$ expression had been correlated with the survival of patients with four neoplasms, the author concluded that deregulation of angiotensinogen and des(angiotensin I)angiotensinogen is associated with survival outcomes in patients with gastric and breast cancer through their anti-angiogenic activity, as opposed to the pro-angiogenic action of angiotensin II with angiotensin II-receptor.

Returning from oncology to cardiovascular diseases, this next publication reestablishes the role of $A G T$ variants and their haplotypes in the etiology of arterial hypertension and therefore 
deserves attention [7]. The subject of interest in a study by Purkait et al. [7] performed in an Indian population were nine SNPs, both in 5' untranslated regions (5'UTR), in exon and in intron regions of $A G T$. Four of them (rs 11568020 , rs5050 - in promoter and rs4762, rs699 — in exon 2) showed a positive association with arterial hypertension $(\mathrm{p}<0.05)$, when 256 hypertensive cases were compared with 158 normotensive controls. Additionally, from the pool of 13 analyzed haplotypes, 3 of them with 2-4/9 minor allele revealed in this study, a pro-hypertensive effect and 1 of them with all major allele - protective association with hypertension $(\mathrm{p}<0.01)$. On the other hand, using multiple logistic regression analysis Khatami et al. [8] found a significant association of the two mentioned in the previous study SNPs — rs4762 and rs699 with ischemic heart disease when in a dominant model 148 Iranian patients with coronary artery disease (100\% hypertensive as described in the patient characteristics) were compared with 135 normotensive controls without coronary artery disease - OR $1.91 ; 95 \%$ CI $1.16-3.15 ; \mathrm{p}=0.01$ and OR $1.8 ; 95 \%$ CI $1.1-2.93$; $\mathrm{p}=0.01$, respectively [8]. The rs4762 and rs699 were also among SNPs which underwent analysis for the purpose of verifying a hypothesis about their linkage with diabetic nephropathy. Mutated alleles of these SNPs presented an association with diabetic nephropathy with OR 10.25; $\mathrm{p}=0.001$ and OR 22.21; $\mathrm{p}<0.001$, respectively, in a comparison of 47 Tunisian type 2 diabetic patients with nephropathy and 189 without this complication [9]. A cohort of 2872 white Australians from the Victorian Family Heart Study were enrolled in a program to test the association of systolic blood pressure with 88 SNPs in AGT, AGTR1, REN and aldosterone synthase gen (CYP11B2). This study by Scurrah et al. [10] revealed that in this group rs2468523 and rs2478544 at $A G T$ presented sexspecific association (not in women), and the presence of every minor allele resulted in an increase of systolic blood pressure in men by 1.58 and $1.63 \mathrm{mmHg}$, respectively [10]. The next paper by Wu et al. [11] presents the problem of occupational exposure on lead, of lead-related hypertension and its association with rs7079 SNP. Although rs7079 nucleotide is located in the untranslated region of the gene it is suggested that this DNA fragment is crucial for binding microRNA and that in this way influences $A G T$ transcription and translation, and subsequently its serum concentration. The authors compared the frequency of minor allele and allele distribution between persons with a blood lead level $<200 \mu \mathrm{g} / \mathrm{L}$ and those with a blood lead level $\geq 400 \mu \mathrm{g} / \mathrm{L}$. Heterozygotic variant (CA) and homozygotic with minor allele (AA) variant of rs7079 appeared more frequently in lead exposed than in unexposed individuals $47.4 \%$ vs. $33.3 \%$; $\mathrm{p}=0.02$. Apart from this, homozygotes with major alleles (CC) had significantly higher blood concentration of angiotensinogen than heterozygotes $(\mathrm{p}=0.01)$. This may be explained by observation that A allele decreased the binding between miRNA and the angiotensinogen gene.

\section{The ACE gene panel}

In recent years some studies on $A C E$ were focused on the association of its SNPs with an inflammatory process. It is reported in many studies that $A C E$ has been associated with sarcoidosis. Although the group of Lahtela et al. [12, 13] did not confirm this link in their own study on Finnish patients, they indicated an interesting trait in the association of rs9905945 with prognosis in this disease. Using generally accepted criteria of World Association of Sarcoidosis and Other Granulomas (WASOG) they divided 188 patients from pulmonary departments into two groups depending on the effects of treatment during a 2-year period: 89 patients in whom the disease resolved and 97 in whom it persisted. They discovered that the combination of rs 9905945 in $A C E$ and of previously reported HLA markers is helpful in predicting the course of this disease in a Finnish population more accurately than previously known. The $\mathrm{C}$ phenotype was statistically more frequent in the better prognosis group than the group with poor prognoses $(79.8 \%$ vs. $66 \%$; $p=0.035)$, and combining the frequencies of $\mathrm{C}$ phenotype with two defined HLA markers revealed a strong association with resolved versus persistent disease prognosis: $37.1 \%$ vs. $11.3 \%$; p < 0.001; OR 4.61 (95\% CI 2.15-9.86). Another interesting association of lung disease with $A C E$ was tested in a population of Chinese children infected with Mycoplasma pneumoniae [14]. Polymorphism within $A C E$ - rs 4340 was analyzed together with SNPs within interleukin-6 gene $(I L-6)-$ rs 1800795 , and within nitric oxide synthase gene (NOS3) — rs1799983 and two other genes. The gene-gene interactions were tested using Multifactor Dimensionality Reduction (MDR) and cumulative genetic risk score approaches. The results of 715 blood samples ( 415 cases of mycoplasmatic pneumonia and 300 healthy controls) showed that a combination of a major allele in $A C E$ (D) with a minor allele in NOS3 (T) contribute to the genetic susceptibility of this germ-related 
pneumonia in children in China: $\mathrm{p}=1.86 \times 10^{-6}$, OR 3.44 (95\% CI 2.014-5.888).

ACE SNPs were also studied for associations with cardiovascular entities. A hypothesis that SNP of angiotensin-converting enzyme 2 gene (ACE2, mapped on chromosome X), may be associated with structural atrial fibrillation (AF) was verified in 300 patients with this most frequent supraventricular arrhythmia and 300 arrhythmia-free controls (mean age in both groups $67.6 \pm 12.5$ and $66.1 \pm 12.5$, respectively; $p=0.133$ ). In those Chinese patients the prevalence of arterial hypertension, diabetes mellitus, coronary artery disease, heart failure and nicotine-addiction were similar in the compared groups. However, the $\mathrm{C}$ allele in rs6632677 was more frequent in males, and when males were analyzed separately from females, the presence of the $\mathrm{C}$ allele increased significantly the risk of $\mathrm{AF}-$ OR 1.954 (95\% 1.196-3.192) [15]. Additionally, an interaction was found between ACE2 and AGTR1 in Chinese patients with this arrhythmia in MDR analysis in a 4-locus model (1 locus in ACE2 and 3 loci in AGTR1). A 3-locus model with the same SNP in ACE2 together with SNP in the troponin I-interacting kinase gene (TNNI3K) and calmodulin III gene (CALM3) was used by Kumar et al. [16] for risk prediction of hypertrophic and dilated cardiomyopathy (HCM and DCM). On the basis of the comparison of genotypes in 130 patients with HCM, 161 - with DCM and 236 controls, the authors concluded that these 3 polymorphisms significantly influenced both cardiomyopathy phenotypes. The 3-locus model predicted the risk of $\mathrm{HCM}$ and DCM with a prediction error of $23.4 \%$ and $22.77 \%$, respectively, with $\mathrm{p}=0.03$ and $\mathrm{p}=0.04$, respectively [16].

Quite a new association was described between three SNPs in the ACE gene located at chromosome 17 and the risk of sudden cardiac death (SCD) during a 64-month follow-up of 1852 participants in the EVOLVE study (EValuation Of Cinacalcet Hydrochloride Therapy to Lower CardioVascular Events), who were from two ancestry groups - European and African - and who were qualified for hemodialysis due to chronic kidney disease with secondary hyperthyroidism [17]. Three correlated SNPs - rs4335, rs4343 and rs 4353 - in DNA from European ancestry patients were associated with a $26-27 \%$ reduction of SCD (OR 0.74 [95\% CI 0.56-0.99], $\mathrm{p}=0.004$; OR 0.74 [95\% CI 0.55-0.99], $\mathrm{p}=0.04$; OR 0.73 [95\% CI $0.54-0.98], \mathrm{p}=0.036$, respectively) and only one SNP - rs 4318 - reduced the risk of SCD by $70 \%$ in the African ancestry patients (OR 0.3 [95\% CI
$0.1-0.85], \mathrm{p}=0.03)$. Some ethnic differences in alleles distribution between European and African ancestry patients were found. In the former group, $\mathrm{A}$ is the minor allele in rs4335 and rs4343 had a frequency of 0.47 for both of them; whereas in the latter group it was $\mathrm{G}$ with a frequency of 0.23 . For the third SNP - rs4353 - the same G was a minor allele in both ancestry groups.

Three SNPs in the ACE gene were investigated among 125 genetic variants in a group of 1009 participants to evaluate influence of genetic variant on the $\mathrm{VO}_{2 \text { peak }}$ level (as a measure of cardiorespiratory fitness) in untrained people in China [18]. Only 1 of the 3 assessed SNPs - rs4295 — was associated with $\mathrm{VO}_{\text {2peak }}\left(\beta=0.87 ; \mathrm{p}<2.9 \times 10^{-4}\right)$ and it was responsible for $1.1 \%$ of interindividual variance in $\mathrm{VO}_{2 \text { peak }}$.

A large-scale study (1024 hypertensive Chinese patients and 956 normotensive ones) was focused on the impact of 34 SNPs of the ACE2 gene located on chromosome X. Interestingly, it was determined that five of them (rs1514283, rs4646155, rs4646176, rs2285666, and rs879922) were associated significantly with hypertension in women, but not in men. For instance, in rs 1514283 the $\mathrm{CC}$ genotype and $\mathrm{C}$ allele were more frequent in hypertensive patients than in controls $-2.2 \%$ vs. $0.7 \%$; OR 4.209 ; $95 \%$ CI $1.633-10.851$; $p<0.05$ and $3.7 \%$ vs. $2.2 \% ; \mathrm{p}=0.01$. According to available research, this study reported for the first time that rs4646155 was associated with essential hypertension in women, and homozygotes with minor allele $\mathrm{T}$ were significantly more frequent in patients with hypertension than in controls $-1.9 \%$ vs. $0.7 \%$; $\mathrm{p}=0.01$; OR $3.492 ; 95 \%$ CI 1.324-9.212 [19]. Interestingly enough, in another study, two of the above-mentioned SNPs (rs4646155 and rs4646176) together with rs2074192, rs2106809 were investigated with regard to whether they could influence not only elevated blood pressure, but also their susceptibility to hypertensive left ventricular hypertrophy (LVH) in Chinese patients. These four SNPs were genotyped in 289 patients with arterial hypertension with LVH and 358 controls without hypertrophy. The presence of minor allele $\mathrm{T}$ in rs2074192 and minor allele T in rs2106809 was significantly associated with $\mathrm{LVH}$, with $\mathrm{p}=0.005$ for both and OR 2.094, 95\% CI 1.249-3.512; OR 2.029, 95\% CI 91.235-3.333, respectively [20].

Since angiotensin converting enzyme 2 interacts with spike glycoprotein of the host to facilitate COVID-19 virus entry to the cells, Khalid et al. [21], carried out an in-silico study to determine the effect of SNPs at ACE2 gene on tertiary structure 
of this protein and the impact of these SNPs on binding the virus. Two variants in ACE2 were identified as those which have an influence on the repulsion of ligands of the same negative charge (G405E, W461R), and the third was probably damaging to the protein (F588S). These findings may explain the differences in the course of severe acute respiratory syndrome coronavirus 2 (SARS-CoV-2) infection-related disease during the current pandemic [21].

\section{Angiotensin receptor panel}

Many polymorphisms listed in this panel were described already in previously cited articles, since SNPs of AGTR1 gene were quite often studied together with SNPs of $A C E$ or $A G T$ genes. An exception to this was a study on the association of rs5186 SNP (A1166C) with retinal vein occlusion; the study involved 69 patients and 82 controls. The minor allele $\mathrm{C}$ was significantly more frequent $(17.4 \%$ vs. $1.2 \%, \mathrm{p}=0.0001)$ in individuals with such complications within the ocular venous vasculature, which confirms earlier reports on the association of this SNP with other vascular events [22]. The rs5186 SNP was also studied with regard to its impact on development of diabetic nephropathy and presented positive association with this complication; $\mathrm{p}<0.0001$ [9]. The same SNP was studied in aforementioned EVOLVE trial, which presented a $31 \%$ increase in risk of composite endpoints (defined as death, nonfatal myocardial infarction, unstable angina leading to hospitalization, aggravation of heart failure or event related to peripheral artery disease) in patients qualified for dialysis - OR 1.31 (95\% CI 1.15-1.49), $\mathrm{p}=4.4 \times 10^{-5}$. The molecular importance of this SNP may be explained by the fact, that AGTR1 is co-expressed with miR-155 in many tissues and the latter molecule represses the expression of AGTR1 only in the presence of a major A allele, but not in the presence of a minor C allele [23]. Additionally, as the EVOLVE study proved, the minor $\mathrm{C}$ allele was found more frequently in patients of European ancestry (28\%) than patients of African ancestry (5\%) [17]. Another AGTR1 polymorphism - rs1492099 — was studied with respect to structural AF in a Chinese population group of 300 patients with this arrhythmia and 300 controls [15]. The authors revealed a higher frequency of minor allele $\mathrm{A}$ in AF-group than in controls $14.2 \%$ vs. $8.8 \%, p=0.004$ with a $72.7 \%$ increased risk of arrhythmia for the minor allele - OR 1.727 (95\% CI 1.154-2.487). Of the four SNPs in AGTR1 gene tested in the Victorian Family Study — rs12721297, rs385338, rs 1800766 and rs275649 — only the first one presented an association with decreased values of systolic blood pressure with $\beta$ (SE) $-2.54(0.76)$ $\mathrm{mmHg}$ [10]. Finally, as it was mentioned for $A C E$, some SNPs of $A G T R$ gene played a critical role in breast cancer. One hundred sixty-one women in a North Indian breast cancer cohort were compared with 152 healthy women, among others with respect to AGTR1 - rs5186 (A1166C) and $\mathrm{ACE}$ insertion/deletion (I/D) polymorphism. The results of analysis suggest a significant association of two tested polymorphisms with the risk of breast cancer: individuals harboring $\mathrm{AC}$ or $\mathrm{CC}$ genotype in AGTR1 together with DD genotype for $A C E$ I/D polymorphism - present increased risk of breast cancer with OR 258 (95\% CI 34.2-1944.4, $\mathrm{p}<0.001)$ [24].

\section{The aldosterone panel}

An important rating enzyme in the process of the synthesis of aldosterone from cholesterol is aldosterone synthase (CYP11B2) and genetic variability in its gene is crucial for blood concentration of this hormone. One of the best investigated SNP of CYP11B2 gene is rs1799998 (T344C), but Qian et al. [25], added some new aspects to existing knowledge after a study on 96 adult patients with chronic kidney disease. They confirmed not only that homozygotes with major alleles (TT) have a significantly higher aldosterone concentration when compared with homozygotes with minor alleles $(\mathrm{CC})-247.5 \pm 93.6 \mathrm{pg} / \mathrm{mL}$ vs. $190.0 \pm$ $\pm 81.7 \mathrm{pg} / \mathrm{mL}, \mathrm{p}=0.036$, but also that the median annual decline of the estimated glomerular filtration rate during 1.5 -year observation was significantly higher in the TT group $(5.2 \pm 16.1 \%)$ than in the CC group $(32.8 \pm 82.5 \%)$ with $\mathrm{p}=0.011$ [25]. Additionally, this group aimed to assess the impact of T344C on incidence of cardiovascular events defined as ST-segment elevation myocardial infarction, non-ST-segment elevation myocardial infarction and unstable angina in this group. The distribution of cardiovascular events was significantly different from random, and it was highest in the TT group (25\%), lowest in the CC group (0\%), and intermediate in the CT group (7.8\%), with $\mathrm{p}=0.033$. Lesser known polymorphisms of CYP11B2 were the subject of investigation in 1024 patients with essential hypertension and 956 normotensive controls [26]. Only one of seven SNPs selected for this study — rs542092383 — was found to increase the risk of essential hypertension - OR 3.48 (95\% CI 1.407-8.597), $\mathrm{p}=0.004$. An interesting result was also obtained for the 
next two SNPs - rs73715282 and rs7463212 after adding them to the haplotype analysis with rs542092383. When major alleles in rs73715282 and rs7463212 were accompanied by a minor allele in rs542092383 (this was observed in $1.1 \%$ of patients with essential hypertension and in $0.2 \%$ of normotensive controls) the OR reached 5.729 (95\% CI 1.889-17.371), with $\mathrm{p}$ values $<0.0005$.

\section{Discussion}

Some practitioners may be disappointed by a very weak impact of SNPs on phenotype in some publications mentioned in this review, e.g., significant but small differences in allele frequency between various study groups. It is important to be aware, however, that such is the nature of SNPs, and a stronger association with clinical manifestation is only possible when a higher number of unbeneficial SNP coexists in an analyzed individual. So, with respect to a benefit for public health, it is prudent to identify individuals at high risk of a given disease, hence it was proposed to consider inclusion of so-called polygenic scores in this procedure. Such scores are already determined for the five most common diseases, including coronary artery disease, AF, diabetes mellitus type 2, inflammatory bowel disease and breast cancer [27]. Their potential advantage over the assessment based on clinical risk factors relies on a possibility to assess them directly after birth, so that primary prevention could be started at the earliest stages of the disease. However, a complex, multifactorial disease such as essential hypertension will probably be even more difficult to predict in childhood on the basis of genetic tests than the already studied diseases.

\section{Limitations of the study}

This review has some limitations: first of all, this article was focused only on SNPs. Other polymorphisms like an insertion/deletion polymorphism or the polymorphism of variable number tandem repeats were ignored, although they also belong in genetic studies of proteins involved in the RAAS [28, 29]. Apart of this, epigenetic changes - DNA methylation, histone modifications or translational control with microRNA may alter the RAAS function. Furthermore, to some extent also enzymatic posttranslational modifications of proteins play a role in the function of the RAAS, although predisposition to protein destabilization depends also on nonsynonymous SNP as was explained by Goswami [6].

\section{Conclusions}

In conclusion, individual susceptibility to hypertension and its associations with genetic variability remain a subject of investigation, which also involve the proteins and enzymes not analyzed in this review. Generally, it should be emphasized that many regions of the genome are still unexplored and knowledge about the importance of underestimated regions will be deepened. Especially, the noncoding regions of the described genes deserve detailed research, as they are expected to constitute 35 times more genetic material than the coding regions [30].

\section{Acknowledgments}

The authors would like to thank Mr. Janusz Wróblewski for his linguistic consultation.

\section{Conflict of interest: None declared}

\section{References}

1. Ji LD, Li JY, Yao BB, et al. Are genetic polymorphisms in the renin-angiotensin-aldosterone system associated with essential hypertension? Evidence from genome-wide association studies. J Hum Hypertens. 2017; 31(11): 695-698, doi: 10.1038/ jhh.2017.29, indexed in Pubmed: 28425437.

2. Cunningham JM, Koytiger G, Sorger PK, et al. Biophysical prediction of protein-peptide interactions and signaling networks using machine learning. Nat Methods. 2020; 17(2): 175-183, doi: 10.1038/s41592-019-0687-1, indexed in Pubmed: 31907444.

3. Kelly TN, Li C, Hixson JE, et al. Resequencing study identifies rare renin-angiotensin-aldosterone system variants associated with blood pressure salt-sensitivity: the GenSalt study. Am J Hypertens. 2017; 30(5): 495-501, doi: 10.1093/ajh/hpx004, indexed in Pubmed: 28199472.

4. Yu S, Peng W, Zhang $\mathrm{H}$, et al. The association between maternal and foetal REN gene polymorphisms and preeclampsia/ eclampsia: A hybrid design study. Pregn Hypertens. 2019; 18: 150-155, doi: 10.1016/j.preghy.2019.09.011, indexed in Pubmed: 31622820 .

5. Procopciuc LM, Nemeti G, Buzdugan E, et al. Renin-angiotensin system gene variants and risk of early- and late-onset preeclampsia: A single center case-control study. Pregn Hypertens. 2019; 18: 1-8, doi: 10.1016/j.preghy.2019.08.006, indexed in Pubmed: 31442828 .

6. Goswami AM. Computational analyses prioritize and reveal the deleterious nsSNPs in human angiotensinogen gene. Comput Biol Chem. 2020; 84: 107199, doi: 10.1016/j.compbiolchem.2019.107199, indexed in Pubmed: 31931433.

7. Purkait P, Halder K, Thakur S, et al. Association of angiotensinogen gene SNPs and haplotypes with risk of hypertension in eastern Indian population. Clin Hypertens. 2017; 23: 12, doi: 10.1186/ s40885-017-0069-x, indexed in Pubmed: 28361007.

8. Khatami M, Heidari MM, Haddadzadeh M, et al. Simultaneous Genotyping of the rs4762 and rs699 Polymorphisms in Angiotensinogen Gene and Correlation with Iranian CAD Patients with 
Novel Hexa-primer ARMS-PCR. Iran J Public Health. 2017; 46: 811-819, indexed in Pubmed: 28828324.

9. Moussa A, Triki S, Hamdouni H, et al. Genetic Variation in the Renin-Angiotensin System and Diabetic Nephropathy in the Tunisian Population. Clin Lab. 2017; 63(3): 469-477, doi: 10.7754/ Clin.Lab.2016.160819, indexed in Pubmed: 28271690.

10. Scurrah KJ, Lamantia A, Ellis JA, et al. Familial analysis of epistatic and sex-dependent association of genes of the renin-angiotensin-aldosterone system and blood pressure. Circ Cardiovasc Genet. 2017; 10(3), doi: 10.1161/CIRCGENETICS.116.001595, indexed in Pubmed: 28506960.

11. Wu Yu, Wang M, Zhang J, et al. A new model of the mechanism underlying lead poisoning: SNP in miRNA target region influence the AGT expression level. Hereditas. 2019; 156: 6, doi: 10.1186/s41065-019-0084-x, indexed in Pubmed: 30700972.

12. Lahtela E, Wennerström A, Pietinalho A. ACE gene variant and sarcoidosis in a Finnish population. Sarcoidosis Vasc Diffuse Lung Dis. 2017; 32: 104-114, indexed in Pubmed: 32476831.

13. Lahtela E, Wolin A, Pietinalho A, et al. Disease marker combination enhances patient characterization in the Finnish sarcoidosis patients. Respir Med. 2017; 132: 92-94, doi: 10.1016/j. rmed.2017.09.014, indexed in Pubmed: 29229112.

14. Zhao J, Zhang W, Shen Li, et al. Association of the ACE, GSTM1, IL-6, NOS3, and CYP1A1 polymorphisms with susceptibility of mycoplasma pneumoniae pneumonia in Chinese children. Medicine (Baltimore). 2017; 96(15): e6642, doi: 10.1097/ MD.0000000000006642, indexed in Pubmed: 28403117.

15. Feng W, Sun L, Qu XF. Association of AGTR1 and ACE2 gene polymorphisms with structural atrial fibrillation in a Chinese Han population. Pharmazie. 2017; 72(1): 17-21, doi: 10.1691/ ph.2017.6752, indexed in Pubmed: 29441892.

16. Kumar A, Rani B, Sharma R, et al. ACE2, CALM3 and TNNI3K polymorphisms as potential disease modifiers in hypertrophic and dilated cardiomyopathies. Mol Cell Biochem. 2018; 438(1-2): 167-174, doi: 10.1007/s11010-017-3123-9, indexed in Pubmed: 28744816.

17. Moe SM, Long J, Schwantes-An THL, et al. Angiotensin-related genetic determinants of cardiovascular disease in patients undergoing hemodialysis. Nephrol Dial Transplant. 2019; 34(11): 1924-1931, doi: 10.1093/ndt/gfy191, indexed in Pubmed: 29982608.

18. Del Coso J, Gu Z, Gerile W, et al. Interindividual variation in cardiorespiratory fitness: a candidate gene study in han Chinese people. Genes (Basel). 2020; 11(5): 555, doi: 10.3390/ genes11050555, indexed in Pubmed: 32429201.

19. Zhang Qi, Cong M, Wang N, et al. Association of angiotensin-converting enzyme 2 gene polymorphism and enzymatic activity with essential hypertension in different gender: a case-control study. Medicine (Baltimore). 2018; 97(42): e12917, doi: 10.1097/ MD.0000000000012917, indexed in Pubmed: 30335025.
20. Fan $Z$, Wu G, Yue M, et al. Hypertension and hypertensive left ventricular hypertrophy are associated with ACE2 genetic polymorphism. Life Sci. 2019; 225: 39-45, doi: 10.1016/j. lfs.2019.03.059, indexed in Pubmed: 30917908.

21. Khalid $Z$, Naveed H. Identification of destabilizing SNPs in SARS-CoV-2-ACE2 protein and spike glycoprotein: implications for virus entry mechanisms. J Biomol Struct Dyn. 2020 [Epub ahead of print]: 1-11, doi: 10.1080/07391102.2020.1823885, indexed in Pubmed: 32964802.

22. Christodoulou A, Bagli E, Gazouli M, et al. Genetic polymorphisms associated with the prevalence of retinal vein occlusion in a Greek population. Int Ophthalmol. 2019; 39(11): 2637-2648, doi: 10.1007/s10792-019-01113-9, indexed in Pubmed: 31065901.

23. Sethupathy P, Borel C, Gagnebin M, et al. Human microRNA-155 on chromosome 21 differentially interacts with its polymorphic target in the AGTR1 3' untranslated region: a mechanism for functional single-nucleotide polymorphisms related to phenotypes. Am J Hum Genet. 2007; 81(2): 405-413, doi: 10.1086/519979, indexed in Pubmed: 17668390.

24. Singh A, Srivastava N, Amit S, et al. Association of AGTR1 (A1166C) and ACE (I/D) Polymorphisms with Breast Cancer Risk in North Indian Population. Transl Oncol. 2018; 11(2): 233-242, doi: 10.1016/j.tranon.2017.12.007, indexed in Pubmed: 29413755.

25. Qian J, Zhong J, Yan M, et al. Modulation of aldosterone level by aldosterone synthase promoter polymorphism and association with eGFR decline in patients with chronic kidney disease. Discov Med. 2018; 26: 251-260, indexed in Pubmed: 30695674.

26. Zhang H, Li X, Zhou Li, et al. A novel haplotype of low-frequency variants in the aldosterone synthase gene among northern Han Chinese with essential hypertension. Medicine (Baltimore). 2017; 96(39): e8150, doi: 10.1097/MD.0000000000008150, indexed in Pubmed: 28953657.

27. Kehra AV, Chaffin M, Aragam KG, et al. Genome-wide polygenic scores for common disease identify individuals with risk equivalent to monogenic mutations. Nat Genetics. 2018; 50: 1219-1224, indexed in Pubmed: 30104762.

28. Say YH. The association of insertions/deletions (INDELs) and variable number tandem repeats (VNTRs) with obesity and its related traits and complications. J Physiol Anthropol. 2017; 36(1): 25, doi: 10.1186/s40101-017-0142-x, indexed in Pubmed: 28615046.

29. Hasimu B, Nakayama T, Mizutani Y, et al. A novel variable number of tandem repeat polymorphism of the renin gene and essential hypertension. Hypertens Res. 2003; 26(6): 473-477, doi: 10.1291/hypres.26.473, indexed in Pubmed: 12862204.

30. Singh KD, Karthikeyan M. Combined sequence and sequencestructure-based methods for analyzing RAAS gene SNPs: a computational approach. J Recept Signal Transduct Res. 2014; 34(6): 513-526, doi: 10.3109/10799893.2014.922575, indexed in Pubmed: 24878201. 